Nervenarzt 2015 $\cdot 86: 1079-1080$

DOI 10.1007/s00115-014-4143-2

Online publiziert: 8. September 2015

(c) Springer-Verlag Berlin Heidelberg 2015
G. Juckel ${ }^{1} \cdot$ M. Bauer ${ }^{2}$

${ }^{1}$ Klinik für Psychiatrie, Psychotherapie und Präventivmedizin,

LWL-Universitätsklinikum Bochum der Ruhr-Universität Bochum

${ }^{2}$ Klinik und Poliklinik für Psychiatrie und Psychotherapie, Universitätsklinikum Carl Gustav Carus, Dresden

\title{
Akut- und Notfallpsychiatrie
}

Die Akut- und Notfallpsychiatrie ist ein Herzstück unseres Fachgebietes der Psychiatrie und Psychotherapie. Wir alle kümmern uns täglich um schwer erkrankte psychiatrische Patienten, aber die Akutpsychiatrie spielt in der Ausbildung unserer Assistenten gerade am Anfang eine ganz entscheidende Rolle, insbesondere um in der Nacht und am Wochenende für die entsprechenden Notfallsituationen gewappnet zu sein. Aber auch viele andere medizinische Disziplinen haben sehr häufig mit psychiatrischen Notfällen zu tun. Es gibt zahlreiche Patienten mit einer psychiatrischen Morbidität oder Komorbidität in der allgemeinen Medizin, in der inneren Medizin oder in der Chirurgie, sodass es doch zu einem beträchtlichen Patientenaufkommen und der entsprechenden Akutsituationen im Notarzteinsatz bzw. in zentralen Notaufnahmen von Kliniken kommt. Hier zu wissen, wie man sich erstens verhält, zweitens wie man sich selber und andere sichert, drittens wie man beruhigend „basal therapeutisch" und später auch psychotherapeutisch auf den Patienten einwirkt und wie man viertens vor allem differenziert eine Pharmakotherapie durchführt, ist für jeden Psychiater, aber auch für viele ärztliche Kollegen der anderen Disziplinen ausgesprochen wichtig.

\section{》) Es besteht großer \\ Bedarf an randomisierten kontrollierten Studien}

Umso mehr erstaunt es, dass für diesen zentralen und auch von der Häufigkeit sehr relevanten Bereich unseres Fach- gebietes kaum systematische und valide Studien vorliegen. Vieles basiert auf Einzelfällen oder Fallserien, sodass allein die Frage einer differenzierten Pharmakotherapie bislang nicht wirklich verlässlich und systematisch untersucht worden ist, sondern im Grunde jede Klinik auf die ihr vertrauten Medikamente „setzt“. Die meisten Kliniken haben zwar entsprechende Empfehlungen für die Akut- und Notfallsituationen, einschließlich einer Fixierordnung, allgemeine Richtlinien oder Leitlinien, die über das einzelne Krankenhaus hinausgehen, sind bislang eher die Ausnahme. Es ist deshalb ein großes Verdienst des Notfall-Referats der Deutschen Gesellschaft für Psychiatrie und Psychotherapie, Psychosomatik und Nervenheilkunde (DGPPN), dass es nicht nur zahlreiche Studien und Befragungen in Gang gesetzt hat, sondern derzeit auch sehr intensiv eine fundierte und evidenzbasierte Leitlinie zur Akut- und Notfallpsychiatrie vorbereitet. In diesem Dokument wird festgestellt werden, dass fundiertes Wissen, insbesondere durch randomisierte kontrollierte Studien, deutlich fehlt und dass wir alle aufgefordert sind, gerade in der Akutsituation noch stärker klinische Forschung zu etablieren. Hier haben wir eine Verantwortung, die weit über das eigene Fachgebiet hinausgeht, damit nach den besten fundierten Erkenntnissen psychiatrischen Notfallsituationen in den medizinischen Einrichtungen begegnet werden kann. Hierfür besteht großer Bedarf, welcher dankenswerterweise vor einiger Zeit in und von der Zeitschrift Deutsches Ärzteblatt ein Stück weit bereits angegangen wurde [1].
Das vorliegende Schwerpunktheft von Der Nervenarzt zur Akut- und Notfallpsychiatrie basiert auf einem Symposium zur „Akutpsychiatrie - ,State of the Art und neue Perspektiven" in Bochum am 06.04.2014 in Zusammenarbeit mit dem DGPPN-Notfall-Referat, auf dem die verschiedenen Aspekte, die jetzt in dieses Schwerpunktheft eingemündet sind, dargestellt und diskutiert wurden.

In dem ersten Beitrag von Prof. Pajonk wird die allgemeine notfall- und akutpsychiatrische Situation in Deutschland dargestellt. Auhand der wenigen Untersuchungen in der Literatur sowie verschiedener eigener Untersuchungen zeigt Kollege Pajonk, dass aufgrund eines psychiatrischen Notfalls jährlich etwa 0,5 Mio. Menschen in Deutschland vom Notarzt und 1,5 Mio. Patienten in der Notaufnahme versorgt werden. Die häufigsten Ursachen sind Intoxikation, Erregungszustände und Suizidalität, wobei in der Regel im höchsten Maße Eigen- und Fremdgefährdung oder andere lebensgefährliche Gesundheitsbedrohungen bestehen. Ein neues notfallpsychiatrisches Thema der letzten Jahre sind vor allem Großschadensereignisse, wie Zugunglücke oder Katastrophen wie der GermanwingsAbsturz. Der Beitrag weist insbesondere darauf hin, dass die psychiatrischen Notfallsituationen in der Häufigkeit vermutlich in den nächsten Jahren weiter zunehmen werden.

Der Beitrag von PD Dr. Neu beschäftigt sich mit der Diagnosestellung und der Differenzialdiagnostik psychiatrischer Akut- und Notfallsituationen. Diese Situationen bringen es mit sich, dass sehr rasch innerhalb von Sekunden und Minuten 
entschieden werden muss, wie das Krankheitsbild einzuschätzen ist, ob möglicherweise eine somatische Verursachung mit zu beachten ist und welche Sofortmaßnahmen zu ergreifen sind. Der Autor verdeutlicht dies anhand mehrerer Fallbeispiele und beschäftigt sich insbesondere mit den akutpsychischen Syndromen der Desorientierung, der Bewusstseinsstörung, der Angst, der Selbstgefährdung, des Mutismus und des Stupors. Entscheidend sei es, die wichtigsten Leitsymptome gut zu kennen, sowohl für den Psychiater als auch für jeden anderen Arzt, um möglichst rasch auf die richtige diagnostische Fährte und damit auf die richtige Behandlung zu kommen [2].

In dem nächsten Beitrag von $P D D r$. Messer und Kollegen werden ausführlich die Allgemeinprinzipien der Pharmakotherapie in der psychiatrischen Akut- und Notfallsituationen dargestellt. Besonderer Verdienst der Autoren ist es, dass sie sehr differenziert auf die einzelnen akut- und notfallpsychiatrischen Situationen eingehen, pharmakotherapeutische Möglichkeiten erörtern und dies in detaillierten und praxisrelevanten Tabellen darstellen. Wichtig ist der Hinweis, dass nicht die Sedierung, sondern die Beruhigung (,„Transquilisation") im Vordergrund steht. Der Patient soll nicht in „Narkose“ versetzt werden, sondern weiterhin gut ansprechbar bleiben, sodass er eben auch entsprechend gesprächs- und psychotherapeutisch behandelt und betreut werden kann. Mittlerweile steht eine ganze Reihe von Medikamenten, insbesondere aus dem Bereich der Antipsychotika und Benzodiazepine, zur Verfügung, die auf verschiedenen Applikationswegen von oral, intravenös, intramuskulär bis hin zu inhalativ eingesetzt werden können. Der Beitrag geht insbesondere auf die Pharmakotherapie von Unruhezuständen, Delir, Katatonien, Stupor sowie Angst und Panik ein und hebt auch medikamentös induzierte Notfälle hervor und erläutert, was hier diagnostisch zu beachten ist und von der Behandlung her getan werden kann und muss.

Der Beitrag von Frau Dr. Mavrogiorgou und Prof. Juckel vertieft das Thema „Erregungszustände“, die eine unmittelbare Intervention und Behandlung verlangen. Zahlreiche Gesichtspunkte bezüg- lich einer organischen Genese oder Intoxikation müssen dabei differenzialdiagnostisch betrachtet werden. Die Behandlung von Erregungszuständen stellt hohe Anforderungen nicht nur an das psychiatrische Wissen, sondern auch an die Persönlichkeit und das Verhalten des Arztes. Oftmals ist ein körperlicher Einsatz notwendig und es ist sehr wichtig, Prinzipien auch der verbalen und nonverbalen Deeskalation sowie klare Handlungsabläufe für sich selber und für das Team zu kennen. Durch Anwendung dieser sowie der heute vorhandenen medikamentösen Behandlungsstrategien kommt es in nahezu allen Situationen mit Erregungszuständen zum raschen Abklingen der akuten Symptomatik.

In dem abschließenden Beitrag von Prof. Wolfersdorf und Kollegen zum Thema "Suizidalität: ein psychiatrischer Notfall - Suizidprävention: eine psychiatrische Verpflichtung" werden die wichtigsten Aspekte suizidaler Syndrome von ihrer Begrifflichkeit, ihrer geschichtlichen Bedeutung sowie in ihrer innerpsychischen Entwicklung sowie Diagnostik und Behandlung dargestellt. Suizidales Verhalten ist weiterhin ein häufiges und schwerwiegendes Problem in Deutschland und in vielen anderen Gesellschaften der Welt. Über 90\% der Menschen mit suizidalem Verhalten haben eine psychische Erkrankung oder entwickeln diese gerade. Das suizidale Syndrom ist in der Regel der höchste Verzweiflungsmoment in der individuellen Existenz eines Menschen und muss unsererseits in der Differenzialdiagnostik und dem therapeutischen Vorgehen sehr ernst genommen werden. Besonderer Verdienst der Autoren ist es, hier insbesondere auf suizidpräventive Maßnahmen und ihre Einsatzmöglichkeiten hinzuweisen, hier liegt auch zukünftig die zentrale psychiatrisch-psychotherapeutische Aufgabe.

Wir hoffen, mit diesen Beiträgen für zahlreiche Leser, insbesondere den jüngeren, ein interessantes Schwerpunktheft zusammengestellt zu haben. Es ist der Stand des Wissens heute, wobei betont sei -wie schon ausgeführt -, dass es in diesem Feld noch viel zu erarbeiten gibt, insbesondere bezüglich klinischer Studien, aber auch der Erstellung bundesweit geltender Richtlinien und Leitlinien - auch für unsere ärztlichen Kollegen in den anderen medizinischen Disziplinen -, sodass zukünftig eine noch bessere akutund notfallpsychiatrische Versorgung der uns anvertrauten Patienten möglich sein wird.

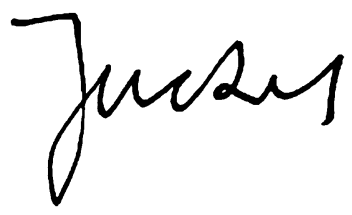

Prof. Dr. Georg Juckel

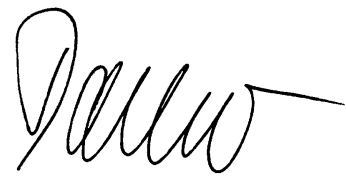

Prof. Dr. Dr. Michael Bauer

\section{Korrespondenzadresse}

\section{Prof. Dr. G. Juckel}

Klinik für Psychiatrie, Psychotherapie und Präventivmedizin, LWL-Universitätsklinikum Bochum der Ruhr-Universität Bochum Alexandrinenstr. 1, 44791 Bochum Georg.juckel@rub.de

Interessenkonflikt. G. Juckel und M. Bauer geben an, dass kein Interessenkonflikt besteht.

\section{Literatur}

1. Mavrogiorgou P, Brüne M, Juckel G (2011) Ärztlich-therapeutisches Vorgehen bei psychiatrischen Notfällen. DÄB 108:222-230

2. Neu P (2011) Akutpsychiatrie. 2. Aufl. Schattauer - Verlag für Medizin und Naturwissenschaften, Stuttgart 